\title{
Experimental study of the operating modes of the resonant current-limiting device
}

\author{
Dmitry V. Mikheev ${ }^{1, *}$, Yelena N. Ryzhkova ${ }^{1}$, Alex $V$. Udaratin $^{2}$, and Regina Salikhova ${ }^{3}$ \\ ${ }^{1}$ National Research University «MPEI», Department of Electric Power Supply of Industrial Enterprises and Electrotechnologies, 111250 \\ Krasnokazarmennaya St. 14, Moscow, Russia \\ ${ }^{2}$ Vologda State University, Institute of Mathematics, Natural and Computer Science, 160000 S. Orlov St. 6, Vologda, Russia \\ ${ }^{3}$ Kazan State Power Engineering University, str. Krasnoselskaya, 51, 420066, Kazan, Russia
}

\begin{abstract}
Decreasing of short-circuit currents in power supply systems enables the usage of less expensive switching-protective devices with a lower breaking capacity and reduction of damage from the consequences of emergency events. In electrical networks of low, medium and high voltage classes, resonant current-limiting devices are used to solve this problem, along with other technical solutions. However, these devices have unsatisfactory weight and dimensions, high cost and other disadvantages. The technical and economic indicators of such devices can be improved through the use of a coil-capacitor (coilcap). Coilcap is a passive element of an electric circuit, which simultaneously possesses inductivecapacitive properties and performs the functions of a reactor and a capacitor in a single technical object. This paper presents a functional diagram of the implementation, design, operating principle and mathematical description of a resonant current-limiting device based on a coilcap. Physical modeling of the steady-state modes of the device (normal mode and current limiting mode) was carried out, and the possibility of limiting short-circuit currents due to the use of a coilcap was confirmed. The practical application of a resonant current-limiting device based on a coilcap can be effectively combined with switching-protective and current-limiting disconnecting devices, relay protection, and automation equipment.
\end{abstract}

\section{Introduction}

The issues of ensuring reliability, stable operation and high efficiency of various objects of power engineering facilities are of high priority today [1-5]. For example, the usual task of power supply systems with quiescent load is to reduce the short-circuit currents. This allows one to select the elements of the electrical network, designed for lower thermal and dynamic loads, to extend the service life of the switching protection equipment, to reduce capital investment and damage caused by an accident [6-8].

One of the options to solve this problem is the use of resonant current limiting devices (CLD), consisting of a reactor, a capacitor bank, and a high-speed saturation choke [6]. However, such devices have several significant drawbacks: unsatisfactory weight and dimensions, high cost, unreliability, and the presence of a nonlinear element.

As an alternative solution, a coil-capacitor (coilcap) can be used in resonant CLD. Coilcap is an electrical device that has both inductive and capacitive properties and has two working parameters: inductance and capacitance [9-11]. Combining the functions of a reactor and a capacitor in a single technical object allows reducing its weight, dimensions, and cost [12].
In this work, we experimentally study the steadystate operating modes of a resonant coilcap-based CLD with limited short-circuit currents.

\section{Design and mathematical model of the resonant CLD based on a coilcap}

Fig. 1 shows a conditional schematic connection diagram of CLD elements based on a coilcap with a connected power supply source $E N$, switch $Q F$ and a load with complex resistance $Z_{\text {load. }}$ The CLD contains a magnetic core $M$ made of electrical steel with a non-magnetic gap $N G$ and correction coils $C C$ to reduce non-linearity and adjust the device parameters and resonance frequencies. Two conductors 1 and 2 are wound on the magnetic core $M$, made in the form of double-lead windings of conductive foil. They are separated from each other by dielectric films $D$. Each conductor has terminals for connecting to each other and the source: conductor 1 has terminals $S_{1}$ and $E_{1}$; conductor 2 has terminals $S_{2}$ and $E_{2}$. A spark gap $S G$ is located between the $E_{1}$ terminal of the conductor 1 and the $S_{2}$ terminal of the conductor 2 . A load is connected to the power supply source $E N$ and to terminal $E_{2}$ of conductor 2 .

* Corresponding author: mikheevdv@ mpei.ru 


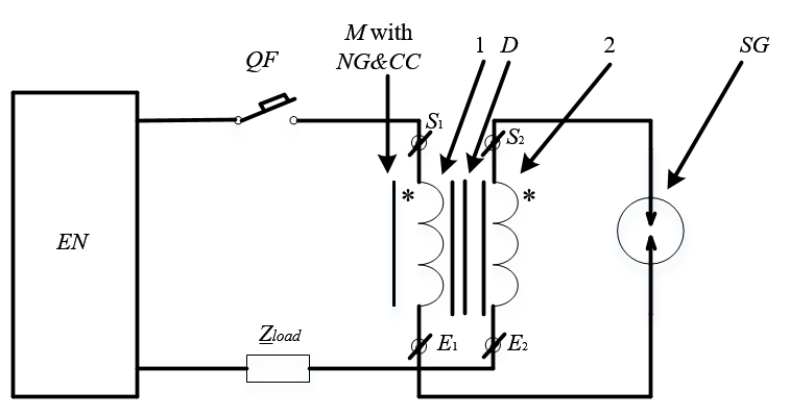

Fig. 1. Conventional schematic diagram of connections between elements of CLD based on coilcap [13,14].

Figures $2 \mathrm{a}$ and $2 \mathrm{~b}$ show a functional block diagram of a two-section coilcap CLD as described above and a photograph of the device physical model. Fig. 3 shows an electric circuit diagram with distributed parameters, the basic connection of CLD elements with respect to terminals $S_{1}, E_{2}$ and terminals $E_{1}, S_{2}$ of conductors 1 and 2 . Here the magnetic circuit $M$, conductors 1 and 2 , dielectric films $D$ are indicated by elements of individual cells of the distributed circuit:

- $R_{0}(\mathrm{Ohm} / \mathrm{m})$ is the active resistance of conductors per unit length;

- $L_{0}(\mathrm{H} / \mathrm{m})$ is the inductance per unit length;

- $C_{0}(\mathrm{~F} / \mathrm{m})$ is the capacitance between conductors per unit length;

- $G_{0}(\mathrm{~S} / \mathrm{m})$ is the active conductivity per unit length.

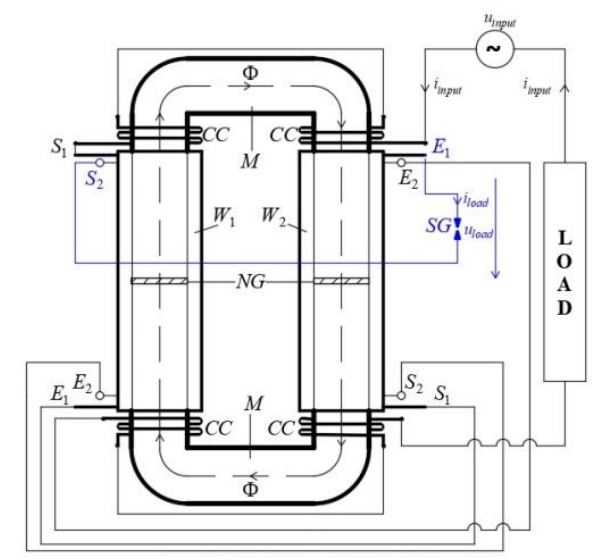

a

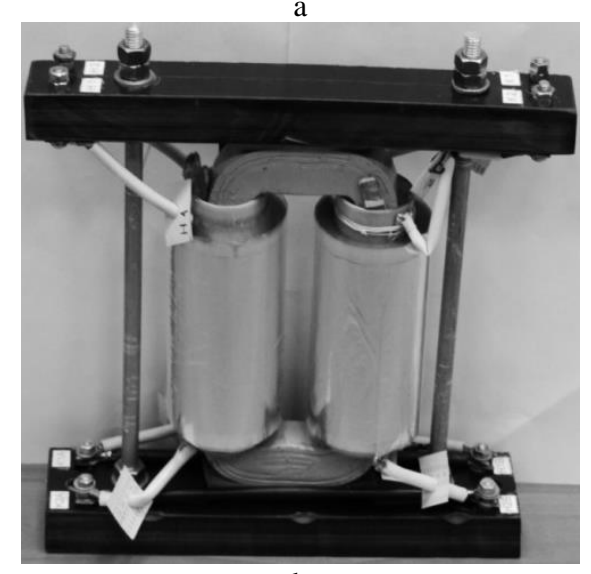

Fig. 2. Functional diagram of CLD based on coilcap (a) and photograph of physical model of coilcap $(b)$ [14-16].

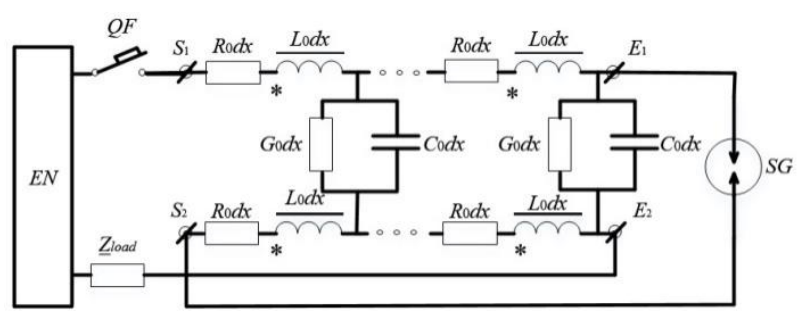

Fig. 3. Electrical equivalent circuit for CLD [4, 7].

Figs. $4 \mathrm{a}$ and $4 \mathrm{~b}$ show the CLD circuits with an equivalent representation of the electrical circuit relative to the device input between the terminal $S_{1}$ of the conductor 1 and the terminal $E_{2}$ of the conductor 2 . The equivalent parts of the equivalent circuits, taking into account the magnetic circuit $M$, conductors 1,2 and dielectric films $D$, are obtained based on the circuit synthesis with distributed parameters [11]. It takes into account that the spark gap $S G$ connected to the $E_{1}$ terminal of the conductor 1 and to the $S_{2}$ terminal of the conductor 2 is open (Fig. 4a) and closed (Fig. 4b).
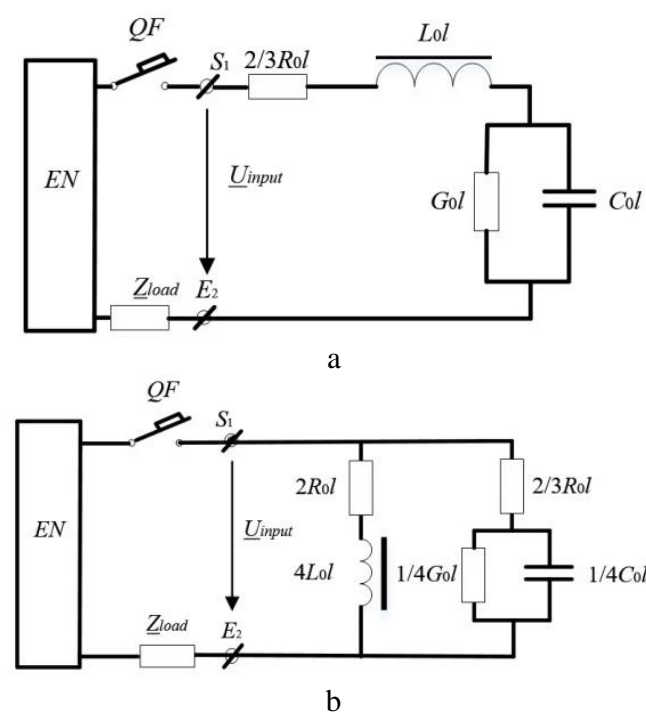

Fig. 4. Equivalent circuit diagram of CLD with open (a) and closed (b) spark gap SG [11,12,14].

\section{Operating principle of the resonant CLD based on coilcap}

\subsection{Normal operation}

In the initial position, according to the device diagram (Fig. 4a), the $Q F$ switch is closed, the $S G$ spark gap is open. Under the action of voltage of the power supply source $E N$ in the circuit containing the switch $Q F$, CLD and the load with the complex resistance $\underline{Z}_{\text {load }}$ the current $I$ is generated.

The voltage resonance takes place at the resonant frequency $f_{0}$. Its value is determined by the electrical capacitance induced by the dielectric films $D$ and conductors 1 and 2 , and the inductance induced by conductors 1 and 2 and the magnetic circuit $M$ with a non-magnetic gap $N G$. The resistance relative to the input of the $\underline{Z}_{\text {input } 1}$ device with an open spark gap $S G$ 
between the $S_{1}$ terminal of the conductor 1 and the $E_{2}$ terminal of the conductor 2 is much less than the load resistance $\underline{Z}_{\text {load }}\left(\underline{Z}_{\text {input } 1}<<\underline{Z}_{\text {load }}\right)$. In accordance with the equivalent representation of the circuit diagram of the device (Fig. 4a), the resistance $\left.\underline{Z}_{\text {input } 1}\right|_{f 0}$ relative to the terminals $S_{1}$ and $E_{2}$ at the resonant frequency $f_{0}$ can be calculated in accordance with [15]:

$$
\left.\underline{Z}_{\text {input } 1}\right|_{f=f_{0}}=\frac{2}{3} \cdot R_{0} \cdot l+G_{0} \cdot l \cdot \frac{L_{0}}{C_{0}}, \text { Ohm. }
$$

From the condition $\operatorname{Im}\left\{\underline{Z}_{\text {input } 1}\right\}=0$ the resonance frequency is $[11,15]$ :

$$
f_{0}=\frac{1}{2 \pi} \cdot \sqrt{\frac{1}{L_{0} \cdot C_{0} \cdot l^{2}}-\frac{G_{0}^{2}}{C_{0}^{2}}}, \mathrm{~Hz} .
$$

In the initial position, when the voltage at the input of the device $\underline{U}_{\text {input }}$ is much less than the load voltage, the voltage at the spark gap $S G$ (at the output of the device), is about 2Q times higher than the input voltage [15], but is not enough for breakdown, and the spark gap $S G$ remains open. The quality factor $Q$, taking into account the neglect of losses in the dielectric due to their smallness $\left(G_{0} \approx 0\right)$, is determined in accordance with [15]:

$$
Q \approx \sqrt{\frac{L_{0}}{C_{0}}} /\left(\frac{2}{3} \cdot R_{0} \cdot l\right) .
$$

\subsection{Operation as a current limiter}

The occurrence of a short circuit is caused by a sharp decrease in the load voltage $\underline{U}_{\text {load }}\left(\underline{U}_{\text {load }} \rightarrow 0\right)$. The current in the loop containing the switch $Q F$ and $C L D$ with open spark gap $S G$ increases significantly. This significantly increases the voltage at the CLD input $\underline{U}_{\text {input }}$ relative to the $S_{1}$ and $E_{2}$ terminals. Considering that the voltage at the device output (the voltage across the open spark gap $S G$ ) is about $2 \mathrm{Q}$ times higher than the voltage at the input of the device, the spark gap $S G$ breaks down, and it closes, which corresponds to the circuit shown in Fig. 4b. Its closure leads to a resonance of currents at the CLD input and an increase in the resistance at the input of the device $\underline{Z}_{\text {input } 1} \rightarrow \underline{Z}_{\text {input } 2}$, which significantly exceeds the load resistance $\underline{Z}_{\text {load }}\left(Z_{\text {input } 2}>>Z_{\text {load }}\right)$. As a result, shortcircuit current is limited in the electrical circuit. The resonance frequencies at resonance of voltages (spark gap is open) (Fig. 4a) and at resonance of currents (spark gap is closed) (Fig. 4b) practically coincide [15].

\section{Experimental study of the steady- state mode}

\subsection{Physical model of a coil-capacitor}

Fig. $2 \mathrm{~b}$ shows a photograph of the physical CLD model based on coilcap. Sections $W_{1}$ and $W_{2}$ are made of two identical sheets of aluminum foil (the number of turns of each section is 150 , foil thickness is $7 \mu \mathrm{m}$, width is 84 $\mathrm{mm})$. They are isolated from each other by two polypropylene films (thickness is $10 \mu \mathrm{m}$, width is 95 $\mathrm{mm})$. The sections are placed on U-shaped sections of the magnetic circuit $M$ (71 KNSR amorphous steel, section of $20 \times 20 \mathrm{~mm}^{2}$ ), which are separated by nonmagnetic gaps $N G$ made of electrical cardboard.

The equivalent lumped parameters of the coilcap equivalent circuit synthesized with respect to the input impedance at $N G=0.6 \times 2=1.2 \mathrm{~mm}$ are the following: $R=R_{0} \cdot l=6.9 \mathrm{Ohm}, L=L_{0} \cdot l=58.52 \cdot 10^{-3} \mathrm{H}, C=C_{0} \cdot l=5.82 \cdot 10^{-}$ ${ }^{6} \mathrm{~F}, G_{C}=G_{0} \cdot l=10 \cdot 10^{-6} \mathrm{~S}$.

\subsection{Description of the laboratory equipment}

Physical modelling of steady-state modes of CLD operation was carried out on a certified computerized laboratory bench of Scientific and Production Enterprise "Educational Technique - Profi" with a software and hardware complex DeltaProfi [17]. It includes power supply sources, blocks of passive elements, measuring instruments, a double-beam oscilloscope, an analogueto-digital converter with 4 voltage sensors $\left(\mathrm{V}_{1}-\mathrm{V}_{4}\right)$ and 4 current sensors $\left(\mathrm{A}_{1}-\mathrm{A}_{4}\right)$, connected via a USB port to a personal computer.

\subsection{Experimental study}

According to Fig. 4a, an electrical circuit was assembled, which included a sinusoidal voltage source $\left(U_{1}=8 \mathrm{~V}\right.$, frequency $f=276 \mathrm{~Hz}$ ), a line with a complex resistance $\underline{Z}_{2}=10+j 17.33 \mathrm{Ohm}$, a load with a complex resistance $\underline{Z}_{4}=100+j 173.3 \mathrm{Ohm}$, physical model of the coilcap with an open spark gap (Fig. 2b) (terminals $S_{1}$ of section $W_{1}$ and $E_{2}$ of section $W_{2}$ are connected to the line and load, terminals $S_{2}$ of section $W_{1}$ and $E_{1}$ of section $W_{2}$ are open). The electrical circuit diagram with connected voltage and current sensors $\left(V_{1}-V_{4}\right.$ and $\left.A_{1}\right)$ is shown in Fig. 5.

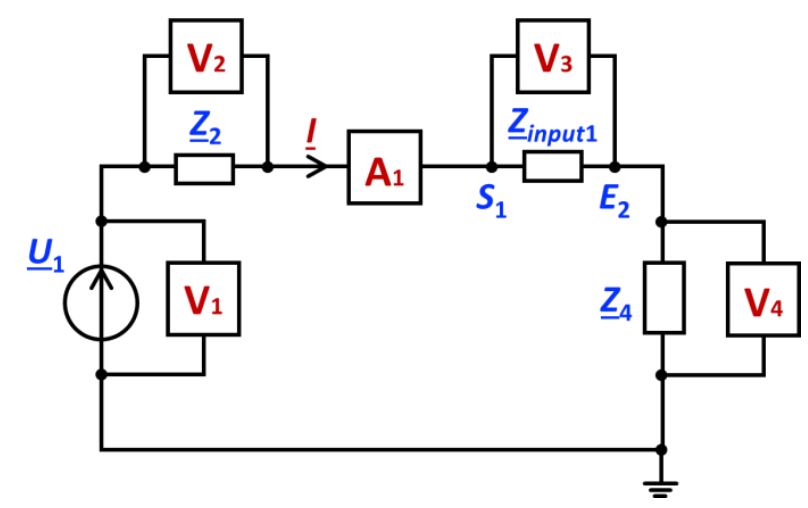

Fig. 5. Electrical circuit diagram in normal operation (DeltaProfi)

In normal operation, a voltage resonance was observed at the input of the physical model of the coilcap ( $I=36 \mathrm{~mA}$ ). Thus, coilcap in this mode was represented by a purely resistive resistance $Z_{\text {input } 1}=R_{\text {input } 1} \approx 4.6 \mathrm{Ohm}$.

Subsequently, a simulation of the current limiting mode (Fig. 6a) was carried out by short-circuiting the load $\left(U_{4}=0 \mathrm{~V}\right)$. During short circuit, the effective current 
$I$ increased to $328 \mathrm{~mA}$ and the voltage $U_{3}$ at the input of the physical model increased from $0.16 \mathrm{~V}$ to $1.43 \mathrm{~V}$. At the same time the voltage on the element modelling a line $U_{2}$ increased from 0.71 to $6.32 \mathrm{~V}$. To limit the shortcircuit current, the coilcap boundary conditions were changed (terminals $S_{2}$ of section $W_{1}$ and $E_{1}$ of section $W_{2}$ were short-circuited by a mechanical contactor), which is shown in Fig. $6 \mathrm{~b}\left(\underline{Z}_{\text {input } 1} \rightarrow \underline{Z}_{\text {input } 2}\right)$. As a result, the input impedance of the physical model of the coilcap increased from $4.6 \mathrm{Ohm}$ to $2.67 \mathrm{kOhm}$, and the current in the electrical circuit decreased from $328 \mathrm{~mA}$ to $3 \mathrm{~mA}$.

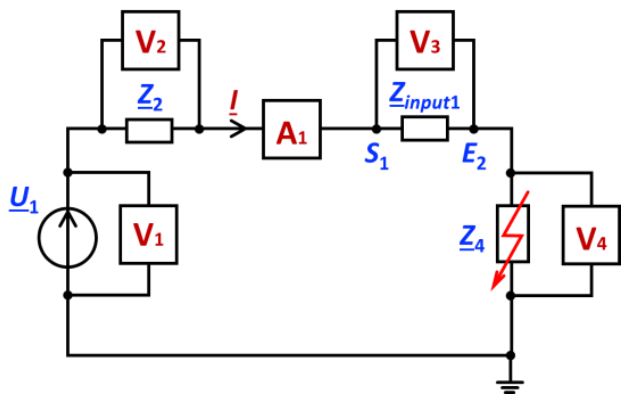

a

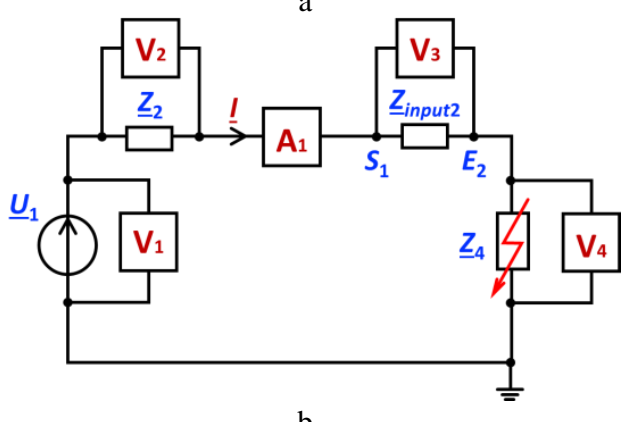

b

Fig. 6. Electric circuit diagram in current limiting mode ( $a-$ $\underline{Z}_{\text {input } 1} ; b-\underline{Z}_{\text {input } 1} \rightarrow \underline{Z}_{\text {input } 2}$ ).

Table 1 shows the measurement results (root-meansquare values of current, voltage and initial phase difference) for normal operation and current limiting mode.

Table 1. The results of modelling the steady-state operation modes of CLD based on coilcap.

\begin{tabular}{|c|c|c|c|}
\hline \multirow{2}{*}{ Characteristic } & \multirow{2}{*}{$\begin{array}{c}\text { Normal } \\
\text { operation }\end{array}$} & \multicolumn{2}{|c|}{ Current limiting mode } \\
\cline { 3 - 4 } & 8 & $\underline{\boldsymbol{Z}}_{\text {input } \mathbf{1}}$ & $\underline{\boldsymbol{Z}}_{\text {input } \mathbf{1} \rightarrow} \underline{\boldsymbol{Z}}_{\text {input } 2}$ \\
\hline$U_{1}, \mathrm{~V}\left(V_{1}\right)$ & 7.14 & 8 \\
\hline$I, \mathrm{~mA}\left(A_{1}\right)$ & 36 & 328 & 3 \\
\hline$U_{2}, \mathrm{~V}\left(V_{2}\right)$ & 0.71 & 6.32 & 0.22 \\
\hline$U_{3}, \mathrm{~V}\left(V_{3}\right)$ & 0.16 & 1.43 & 8 \\
\hline$U_{4}, \mathrm{~V}\left(V_{4}\right)$ & 7.19 & 0 & 0 \\
\hline$\varphi_{U 1-I},{ }^{\circ}$ & 59 & 49 & -4 \\
\hline$\varphi_{U 2-I},{ }^{\circ}$ & 60 & 61 & 63 \\
\hline$\varphi_{U 3-I},{ }^{\circ}$ & 0 & 0 & 0 \\
\hline
\end{tabular}

The results of the physical modelling of the steadystate operation modes of the resonant CLD based on the coilcap indicate the fundamental possibility of solving the problem of limiting the current in the line during a short circuit. The performed theoretical calculations (for the circuits shown in Figs. 4a and 4b) of the steady-state operation modes of the proposed device for limiting short-circuit currents quite accurately coincide with the experimental results (Table 1).

\section{Conclusions}

Physical modelling of steady-state modes of operation of resonant CLD based on coilcap has been carried out. For the first time, the fundamental possibility of using coilcap for limiting short-circuit currents and overload currents has been experimentally confirmed.

It is demonstrated that when solving design problems and analysis of the operating modes of the coilcap-based CLD, simplified equivalent circuits with lumped parameters, synthesized with respect to the input terminals of the device, can be used.

To ensure the full functioning of the proposed CLD, it is necessary to use means of relay protection and automation. In addition, the proposed device can be effectively combined with current-limiting disconnecting devices (for example, fuses and automatic circuit breakers) with a small breaking capacity (for example, in cases when the short-circuit currents exceed their rated breaking capacity).

To reduce active power losses in normal operation and to effectively limit short-circuit currents, it is necessary to ensure a sufficiently high Q-factor of the coilcap in resonant modes (at least 80 ) by choosing a magnetic circuit, conductors, correction coils and a dielectric with a low level of losses.

The use of coilcap allows one to simplify the design of the resonant CLD, to ensure its versatility and to reduce the weight and dimensions by using simultaneously two operating parameters in one device.

\section{Acknowledgements}

This work was supported by the Russian Foundation for Basic Research, project no. 19-08-00730-a.

\section{References}

1. A. Kulachinskaya, I.G. Akhmetova, V.Y. Kulkova, S.B. Ilyashenko, The challenge of the energy sector of russia during the 2020 covid-19 pandemic through the example of the republic of tatarstan: Discussion on the change of open innovation in the energy sector, J. Open Innov. Technol. Mark. Complex., 6 (2020)

2. V. Markov, A. Fedyukhin, I. Sultanguzin, B.G. Matisov, N.V. Nikitkov, V.V Izrantsev, V.G. Knorring, G.A. Konrashkova, L.R. Mukhametova, Improvement of energy efficiency of motor fuel production at oil refinery, IOP Conf. Ser. Earth Environ. Sci., 337 (2019)

3. I.G. Akhmetova, A.A. Kalyutik, A.V. Fedukhin, O.V. Derevianko, L.R. Mukhametova, Determination of additional factors in assessing the reliability of heat supply systems, IOP Conference Series Earth and Environmental Science, 288, 012076 (2019), DOI: $10.1088 / 1755-1315 / 288 / 1 / 012076$ 
4. A.G. Saidkhodjaev, B.K. Ametova, M.M. Mamutov, Intellectualization of determination of electrical loads in city electric networks, E3S Web Conf., 139, 01072 (2019)

5. I.G. Akhmetova, K.V. Lapin, T.R. Akhmetov, E.Y. Balsamova, Digitalization of heat energy accounting as a means of improving the reliability of heat supply, E3S Web Conf., 139, 01011 (2019)

6. T.V. Ancharova, N.N. Guseva, Optimization of short-circuit currents with considering account power quality requirements (Moscow: Publishing house of MPEI, 2017)

7. I.N. Fomin, R.P. Belikov, T.A. Kudinova, A.N. Tsvetkov Identification power line sections with increased-electricity losses using sensors with Wi-Fi technology for data transmission, E3S Web Conf., 178, 01083 (2020)

8. A. Akhmetshin, G. Marin, D. Mendeleev, Modeling of asynchronous motor operation modes for the correct selection of voltage regulation devices, E3S Web Conf., 178, 01015 (2020)

9. R. Reeves, Choke-Capacitor Hybrid As a Fluorescent-Lamp Ballast, Proc. Inst. Electr. Eng., 122, 1151-1152 (1975)

10. I.V. Volkov, S.I. Zakrevskiy, Distributed Parameter Converter for Current Regulation in Variable Load, Elektrichestvo, 10, 40-43 (1984)

11. K.S. Demirchyan, G.G. Gusev, Synthesis of the equivalent circuit of the inductance coil with the Reactive power self-compensation, Izv. Akad. Nauk SSSR, Energ. Transp., 2, 3-10 (1987)

12. P.A.Butyrin, G.G. Gusev, D.V. Mikheev, F.N. Shakirzianov, Algorithm of definition of parameters of Katkon-Element of optimization of electrical networks modes, Therm. Eng., 62, 946-949 (2015)

13. P.A. Butyrin, G.G. Gusev, D.V. Mikheev, V.V. Sirenko, F.N. Shakirzianov, RF Patent 2660177 Short-circuit current limiting device (2018)

14. P.A. Butyrin, G.G. Gusev, D.V. Mikheev, F.N. Shakirzianov, Coil-capacitor as a basis for creating efficient devices for distribution power networks NEIS 2018, Conf. Sustain. Energy Supply Energy Storage Syst. 173-178 (2018)

15. P.A. Butyrin, G.G. Gusev, D.V. Mikheev, F.N. Shakirzianov, Coil Capacitor for an InductiveCapacitive Converter, Bull. Russ. Acad. Sci. Phys., 82, 918-921 (2018)

16. P.A. Butyrin, G.G. Gusev, V.V. Kuzhman, D.V. Mikheev, F.N. Shakirzianov, Mathematical and physical modeling of a filter-compensating device based on a coilcap, Elektrichestvo, 11, 58-62 (2014)

17. L.V. Alexeychik, M.P. Zhokhova, D.V. Mikheev, M.V. Karpunina, Electrotechnical Laboratory: From Physical Experiment to Virtual Scenario 2018 4th International Conference on Information Technologies in Engineering Education, Inforino 2018 - Proceedings (2018) 